\title{
A multipronged approach to understanding the form and function of hStaufen protein
}

\author{
SILVIA VISENTIN, ${ }^{1,2,3}$ GIUSEPPE CANNONE, ${ }^{4,5}$ JAMES DOUTCH ${ }^{3}$ GEMMA HARRIS, ${ }^{6}$ \\ MICHAEL L. GLEGHORN, ${ }^{7}$ LUKE CLIFTON, ${ }^{3}$ BRIAN O. SMITH, ${ }^{1}$ and LAURA SPAGNOLO ${ }^{1}$ \\ ${ }^{1}$ Institute of Molecular Cell and Systems Biology, University of Glasgow, Glasgow G12 80Q, United Kingdom \\ ${ }^{2}$ Institute of Cell Biology, School of Biological Sciences, University of Edinburgh, Edinburgh EH9 3JQ, United Kingdom \\ ${ }^{3}$ ISIS Pulsed Neutron and Muon Source, Science and Technology Facilities Council (STFC), Rutherford Appleton Laboratory, \\ Didcot OX11 OQX, United Kingdom \\ ${ }^{4}$ Institute of Quantitative Biology, Biochemistry and Biotechnology, School of Biological Sciences, University of Edinburgh, Edinburgh EH9 3JQ, \\ United Kingdom \\ ${ }^{5}$ MRC Laboratory of Molecular Biology, Cambridge CB2 0QH, United Kingdom \\ ${ }^{6}$ Research Complex at Harwell, Rutherford Appleton Laboratory, Research Complex at Harwell, Didcot OX11 OFA, United Kingdom \\ ${ }^{7}$ School of Chemistry and Materials Science, College of Science, Rochester Institute of Technology, Rochester, New York 14623, USA
}

\begin{abstract}
Staufen is a dsRNA-binding protein involved in many aspects of RNA regulation, such as mRNA transport, Staufen-mediated $m R N A$ decay and the regulation of mRNA translation. It is a modular protein characterized by the presence of conserved consensus amino acid sequences that fold into double-stranded RNA binding domains (RBDs) as well as degenerated RBDs that are instead involved in protein-protein interactions. The variety of biological processes in which Staufen participates in the cell suggests that this protein associates with many diverse RNA targets, some of which have been identified experimentally. Staufen binding mediates the recruitment of effectors via protein-protein and protein-RNA interactions. The structural determinants of a number of these interactions, as well as the structure of full-length Staufen, remain unknown. Here, we present the first solution structure models for full-length hStaufen $1^{55}$, showing that its domains are arranged as beads-on-a-string connected by flexible linkers. In analogy with other nucleic acid-binding proteins, this could underpin Stau1 functional plasticity.
\end{abstract}

Keywords: SAXS; Staufen; dsRNA-binding domain; structural biology

\section{INTRODUCTION}

Staufen (Stau) is a dsRNA-binding protein originally identified in Drosophila melanogaster, where it plays an essential role in oocyte development (Schupbach and Wieschaus 1986; St Johnston et al. 1991). It is well conserved from nematodes to humans and, depending on the species, is composed of four or five dsRNA-binding domains (RBDs) (Wickham et al. 1999). In humans, there are two Staufen paralogs: hStau1 and hStau2, each present in several isoforms (Park et al. 2013). Much of our knowledge on human Stau 1 is based on the study of isoform hStau $1^{55} \cdot \mathrm{hStau}^{55}$ is associated with 405 and 605 ribosomal subunits and colocalizes with the rough endoplasmic reticulum (Marión et al. 1999; Wickham et al. 1999; Luo et al. 2002). hStau ${ }^{55}$ has also been characterized biochemically in the context of

Corresponding author: Laura.Spagnolo@glasgow.ac.uk

Article is online at http://www.rnajournal.org/cgi/doi/10.1261/rna. 072595.119.
mRNA decay (Kim et al. 2005) and cell cycle control (Boulay et al. 2014). While Stau2 is expressed primarily in the neuromuscular system and is mostly involved in mRNA transport at particular sites of the post-synaptic muscles, Stau1 is ubiquitously expressed (Bélanger et al. 2003; Lebeau et al. 2008; Vessey et al. 2008; Ravel-Chapuis et al. 2012; Peredo et al. 2014). Even though Stau1 and Stau2 exhibit different tissue expression patterns, they have been shown to be involved in the same mechanisms of RNA regulation, such as mRNA transport (Martel et al. 2006, 2010; Ramasamy et al. 2006; Vessey et al. 2008; Ravel-Chapuis et al. 2012), Staufen-mediated mRNA decay (SMD) (Kim et al. 2005, 2014; Gong et al. 2009; Gong and Maquat 2011; Cho et al. 2012; Baker et al. 2013; Park and

\footnotetext{
(C) 2020 Visentin et al. This article is distributed exclusively by the RNA Society for the first 12 months after the full-issue publication date (see http://rnajournal.cshlp.org/site/misc/terms.xhtml). After 12 months, it is available under a Creative Commons License (Attribution-NonCommercial 4.0 International), as described at http:// creativecommons.org/licenses/by-nc/4.0/.
} 
Maquat 2013) and regulation of mRNA translation (RavelChapuis et al. 2012; Bonnet-Magnaval et al. 2016), myogenic differentiation (Ravel-Chapuis et al. 2014), stress granule formation(Ravel-Chapuis et al. 2016), regulation of adipogenesis (Cho et al. 2012; Baker et al. 2013), progression of the cell cycle (Boulay et al. 2014), and cellular differentiation (Gautrey et al. 2005, 2008; Kretz 2013; Peredo et al. 2014). They also are central players in virology, functioning in HIV infection by favoring viral RNA (vRNA) encapsidation (Mouland et al. 2000; Chatel-Chaix et al. 2004, 2008; Banerjee et al. 2014), in hepatitis C infection by transporting $v R N A$ to the site of translation, in the replication of cellular DNA (Blackham and McGarvey 2013; Dixit et al. 2016) and as requirements for efficient influenza A virus propagation (de Lucas et al. 2010). The variety of cellular processes in which Stau1 is implicated suggests that it might adopt different binding modes with its diverse RNA targets and that structurally distinct RNA-Stau 1 complexes mediate the recruitment of effectors via proteinprotein and/or protein-RNA interactions.

Interactions between Stau1 and its RNA substrates were initially characterized for $h \mathrm{Stau} 1^{55}$. Multiple copies of hStau $1^{55}$ can bind a single dsRNA. In cells, Stau 1 binds intramolecular duplexes within the hARF1 mRNA (Martel et al. 2010). Furthermore, in vitro, multiple copies of Stau1 bind to mRNAs containing as many as 250 CUG repeats (Ravel-Chapuis et al. 2012). Additionally, the finding that hStau $1^{55}$ stabilizes imperfectly base-pairings formed between mRNAs and IncRNAs (Gong and Maquat 2011), suggests that multiple hStau1 molecules bind to the same dsRNA. Genome-wide analysis (Furic et al. 2008; Laver et al. 2013) and hiCLIP (RNA hybrid and individualnucleotide resolution ultraviolet cross-linking and immunoprecipitation) (Fernandez Moya and Kiebler 2015; Sugimoto et al. 2015) of Stau-associated mRNAs identified secondary structures that confer binding specificity (Ricci et al. 2014). Nevertheless, what defines a Stau binding site remains unclear (de Lucas et al. 2014).

Stau proteins are characterized by two conserved consensus amino acid sequences that fold into dsRNA-binding domains (RBD3 and RBD4); hStau1 contains two other RBDs (RBD2 and RBD5) that are unable to bind RNA and, relative to hStau1, hStau2 has an additional RBD1 and only a partial RBD5 (Buchner et al. 1999; Wickham et al. 1999; Duchaine et al. 2002; Allison et al. 2004; Furic et al. 2008). hStau1 and hStau2 tubulin-binding domains (TBDs), which are involved in mRNA transport on the cytoskeleton, share only $18 \%$ identity. Functional activation of a number of dsRNA-binding proteins requires that they selfassociate or associate with other dsRNA-binding proteins (Park et al. 2013). A Staufen swapping motif (SSM) has been identified to reside between TBD and RBD5. The SSM is necessary for the homodimeric or heterodimeric interactions between Stau1 and Stau2 (Park et al. 2013). This dimerization is critical for SMD (Martel et al. 2010;
Gleghorn et al. 2013; Park et al. 2013). The amino-terminal $\alpha$-helix of RBD5 was also identified as the major determinant for protein-protein interaction in vivo, intercalating with the two $\alpha$-helices of the SSM. A recent SEC-MALLS report on purified protein also showed that, in the absence of RNA, SSM-RBD5 promotes dimerization (Lazzaretti et al. 2018). The importance of RBD2 in dimerization is less clear. BRET assays, aimed at the study of hStau $1^{55}$ multimerization, show that RBD2 (amino acids 37-79 of isoform hSTAU ${ }^{55}$ ) interacts with full-length hStau1 (Martel et al. 2010). On the other hand, recombinant purified hStau1"RBD"2-RBD3 suggests that the contribution of RBD2 to hStau ${ }^{55}$ dimerization, while existing, is relatively minor (Martel et al. 2010; Park et al. 2013).

To date, analyses of the three-dimensional structure of Stau proteins have focused on studies of truncated versions of the protein, either in isolation or in complex with short RNA sequences or in complex with truncated versions of interacting proteins. The NMR structure of Drosophila RBD3 first confirmed that this construct is organized in the typical $\alpha-\beta-\beta-\beta-\alpha$ fold (PDB ID: 1 STU) (Bycroft et al. 1995). Mouse Stau2 RBD4, in the absence of dsRNA, also showed the $\alpha-\beta-\beta-\beta-\alpha$ fold (PDB ID: $1 \mathrm{UHZ}$ ). The structure of human Stau1 SSM-RBD5 solved by $X-$ ray crystallography revealed a domain swapped dimer, which is responsible for mediating hStau1 dimerization (PDB ID: 4DKK) (Gleghorn et al. 2013). The X-ray crystal structure of the complex between Miranda and RBD5 showed two RBD5s symmetrically bound to the Miranda dimeric coiled-coil region through their exposed $\beta$-sheet faces, revealing a previously unrecognized protein interaction mode for RBDs (PDB ID: 5CFF) (Jia et al. 2015).

The solution structure of Drosophila melanogaster Stau RBD3 bound to a 12-bp stem-loop RNA, determined by NMR spectroscopy, revealed the interaction of the canonical $\alpha-\beta-\beta-\beta-\alpha$ RBD fold with dsRNA (PDB ID: 1EKZ) (Ramos et al. 1999, 2000). The crystallographic structure of the RBD3-RBD4 construct, bound to dsRNA as a dimer (monomers $A$ and $B$ ), shows that the interaction surface with the RNA spans the major groove and the two adjacent minor groove surfaces. Furthermore, RBD3 from monomer B is bound on the opposite side of the RNA molecule, in an antiparallel orientation to RBD3A, whereas density for the sec RBD4 is missing (Lazzaretti et al. 2018). Human, Drosophila, and Caenorhabditis elegans Stau bind dsRNA without apparent sequence specificity in vitro (St Johnston et al. 1992; Marión et al. 1999; Wickham et al. 1999; Ramos et al. 2000; LeGendre et al. 2013; Wang et al. 2015). Bono and coworkers recently showed that, in addition to the interactions with the sugar-phosphate backbone previously identified for RBD3 (Ramos et al. 2000), both domains of hStau1 directly contact RNA bases in the minor groove of the dsRNA used. Indeed, they also show that specific base recognition is relevant in vivo and may therefore contribute to the overall sequence selectivity by Stau, possibly together with 
additional regions of the protein or with other regulators (Lazzaretti et al. 2018). The macromolecular interaction events that happen downstream from Stau RNA binding are still structurally unknown.

We used an integrated structural biology approach, combining homology modeling, small-angle X-ray scattering (SAXS), NMR, and hydrodynamic methods to characterize the structure of the human full-length Staufen 1 protein. To interpret this structure, we also studied an hStau $1^{55} \_$RBD2 truncated variant, individual hStau1 ${ }^{55}$ domains, and several tandem multidomain Stau1 fragments. We confirmed that the deletion of RBD2 influences the oligomeric state of the protein, as well as reporting for the first time its effect on protein solubility. Our data show for the first time that $\mathrm{hStau}{ }^{55}$ adopts an elongated conformation in solution. Furthermore, in the absence of RNA, RBD3 and RBD4 are connected by a linker that is very flexible in solution, and they do not interact with one another.

The reshaping or folding of flexible components in the presence of target nucleic acid in proteins has already been linked to multifunctionality. As an example, DNA and RNA nucleases (Tsutakawa et al. 2014) behave like molecular level transformers that can rebuild themselves by sometimes altering their protein conformations and typically sculpting the nucleic acid to control both their specificity and efficiency functions. We propose that the extreme flexibility and the independent movement of individual domains could also be the basis for the functional plasticity of Stau1 protein: Different relative movements of domains on themselves and/or on RNA can create multiple joint recognition surfaces, reshaping itself to elicit diverse RNA metabolism tasks.

\section{RESULTS AND DISCUSSION}

\section{RBD2 influences both solubility and oligomeric state of Staufen 1}

Stau proteins were purified to homogeneity by immobilized nickel chromatography followed by size exclusion chromatography. Additive screening to determine conditions that would allow the protein to achieve high concentration and good homogeneity for subsequent structural studies was performed using $10 \mathrm{~K}$ MWCO spin-concentrators (Rambo 2017). The addition of L-Arg HCl to the buffer proved necessary for maintaining the solubility of the full-length protein to enable further experiments. Interestingly, the solubility of hStau ${ }^{55} \triangle \mathrm{RBD} 2$ is not affected by the presence (or absence) of L-Arg $\mathrm{HCl}$ in the buffer. However, this additive was used for all constructs for consistency with the purification requirements of hStau $1{ }^{55}$ _FL. Size exclusion chromatography (SEC) traces show that the hydrodynamic volumes of hStau $1^{55}$ FL and of hStau ${ }^{55}$ _RBD2 $(\sim 130-140 \mathrm{kDa})$ are higher than expected for globular proteins with corresponding molecular weights, suggesting that the two proteins might have an elongated shape or might form homomultimers. The domains organization of hStau $1{ }^{55}$ _FL and hStau $1{ }^{55} \_$RBD2 is shown in Figure 1A. SEC-MALS analysis of hStau1 ${ }^{55}$ _FL (Fig. 1B) shows the presence of multiple assemblies. In contrast to the recently published SEC profile for $\mathrm{hStau} 1{ }^{63} \mathrm{FL}$ (Lazzaretti et al. 2018), both SEC profiles for hStau1 ${ }^{55}$ _FL and $h \mathrm{Stau} 1{ }^{55}{ }_{-} \mathrm{RBD} 2$ described in this study present symmetrical peaks. MALS analysis of the eluting species highlights the different behavior of hStau1 ${ }^{55} \mathrm{FL}$ and hStau1 ${ }^{55} \_$RBD2. Measurements were performed at three different concentrations $(20,100$, and $200 \mu \mathrm{M})$. The samples used for this analysis had not been subject to the final SEC purification, hence small amounts of species other than $\mathrm{hStau} 1{ }^{55}$ _FL and hStau $1{ }^{55} \_$RBD2 were seen to be present. However, the predominant peak in the hStau $1^{55} \_$RBD2 sample had a molecular weight consistent with that of a monomer (Fig. 1C). The main SEC peak for hStau $1^{55}$ FL appears with a molecular weight consistent with a dimer but the high polydispersity seen across the peak suggests that this is an equilibrium species between a monomer and higher order oligomers. This is consistent with the previous observation that RBD2 mediates hStau1 ${ }^{55}$ _FL self-association (Martel et al. 2010; Lazzaretti et al. 2018), showing that its presence is fundamental for the formation of a stable oligomer in solution. The recent report describing $\mathrm{hStau} 1^{63}$ did not contain SEC-MALS analysis for the full-length protein (Lazzaretti et al. 2018).

To resolve the oligomeric assemblies of the species eluting in the main SEC peak, we performed analytical ultracentrifugation (AUC) experiments. AUC analysis of the peak fraction from hStau ${ }^{55}$ FL SEC (Fig. 1D) confirms the coexistence of a number of species with molecular weights consistent with that of the monomer (major species in solution) and higher oligomers. Measurements were performed at three different concentrations $(4,25$, and $100 \mu \mathrm{M})$. We chose to analyze a wide range of concentrations to address the role of concentration in the oligomeric state of the protein. The number of oligomers increases with increasing sample concentration, and the position of the peaks also shifts to a higher sedimentation coefficient. Both of these phenomena indicate concentration-dependent self-association equilibrium for $\mathrm{hStau} 1^{55}$ _FL. On the other hand, both SEC-MALS (Fig. 1C) and AUC (Fig. 1E) analysis of hStau $1^{55} \_$RBD2 show that the truncated protein is only present in solution as monomer.

\section{Staufen adopts distinct elongated structures in solution}

The first indication that Staufen protein and its hStau ${ }^{55}$ $\triangle \mathrm{RBD} 2$ mutant adopt elongated structures in solution is given by their average hydrodynamic volume, which is much higher than expected for the estimated molecular weight of the monomeric protein. This is in agreement 


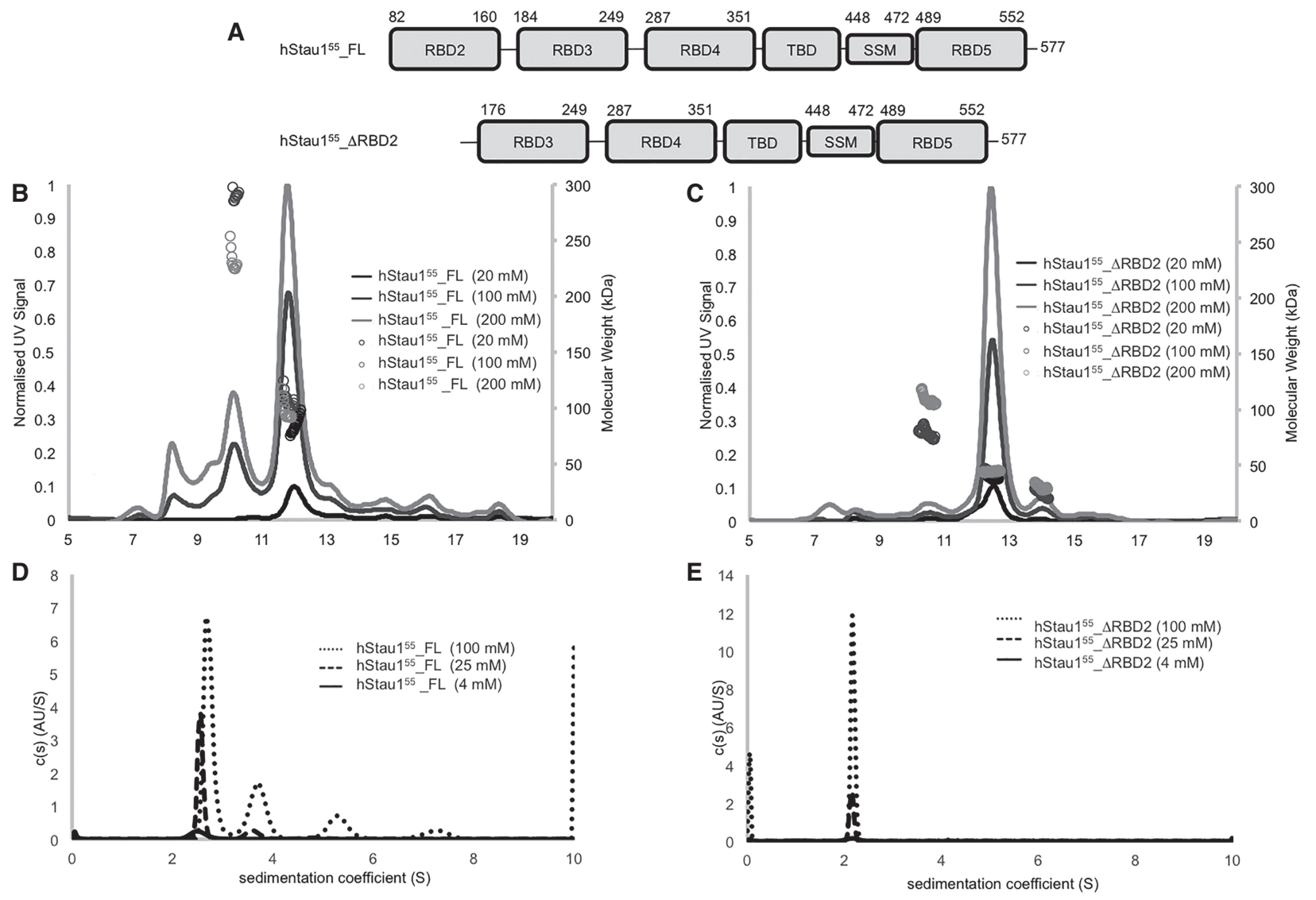

FIGURE 1. Architecture and biophysical characterization of Staufen proteins. (A) Domains organization of hStau $1^{55}{ }_{-} \mathrm{FL}$ and hStau $1^{55} \_\Delta R B D 2$. (B) SEC-MALS of hStau $1^{55}$ FL at 20, 100, and $200 \mu \mathrm{M}$. (C) SEC-MALS of hStau $1^{55} \_$RBD2 at 20, 100, and $200 \mu$ M. (D) Analytical ultracentrifugation (AUC) of hStau1 ${ }^{55}$ FL at 4, 25, and $100 \mu \mathrm{M}$. (E) Analytical ultracentrifugation (AUC) of hStau1 ${ }^{55} \_\Delta \mathrm{RBD} 2$ at 4, 25, and $100 \mu \mathrm{M}$.

with the $R_{\mathrm{g}}$ and $D_{\max }$ obtained from SAXS measurements for both the full-length $\left(R_{\mathrm{g}}\right.$ ensemble $=48.11 \AA, D_{\max }$ ensemble $=155.2 \AA)$ and truncated $\triangle \mathrm{RBD} 2\left(R_{\mathrm{g}}\right.$ ensemble $=$ $50.26 \AA, D_{\max }$ ensemble $=166.96 \AA$ ) proteins. The higher $R_{\mathrm{g}}$ and $D_{\max }$ for hStau1 ${ }^{55} \_\mathrm{RBD} 2$ can be explained by a higher degree of conformational heterogeneity in the FL protein. Importantly, SAXS shows that both these systems are characterized by a high degree of flexibility, as shown by their Kratky plot in Figure 2. The three-dimensional models of hStau $1^{55}$ FL protein and of its truncation mutant hStau $1^{55} \_$RBD2 were obtained by combining homology modeling analysis and SAXS data. The models obtained for $h$ Stau $1^{55} \_$RBD2 show that the protein adopts a range of conformations (Fig. 2), from highly extended to more compact, where RBD3 and RBD5 are in closer proximity. The relative positions of RBD4, TBD, and SSM show only minor differences among the models obtained, due to the flexibility of the loops. On the other hand, the high level of flexibility of the loops between RBD3 and RBD4 and between SSM and RBD5 seems to be the main factor that contributes to the coexistence of a more distended and a more closed conformation of hStau $1{ }^{55} \triangle \mathrm{RBD} 2$. These models show that all the individual domains do not coa- lesce to form a compact structure. The models obtained for hStau $1^{55} \mathrm{FL}$, represented in Figure 3, show more interdomain flexibility, resulting in the presence of elongated, as well as more compact, conformations. The major differences between the coexisting conformations are due to the disordered loops between RBD2 and RBD3, between RBD3 and RBD4 and between RBD4 and TBD. Thanks to the malleability of these linkers, hStau $1{ }^{55}$ FL seems to be able to transition in solution from more elongated to more closed conformations, but still not globular.

The structural information gathered from the SAXS models for hStau1 ${ }^{55}$ _FL and $h S$ tau $1{ }^{55}{ }_{-} \triangle \mathrm{RBD} 2$ were validated using 2D NMR (Fig. 4). ${ }^{15} \mathrm{~N},{ }^{1} \mathrm{H}$-TROSY spectra were recorded for individual and tandem domains, as well as for the fulllength protein and its truncation mutant $\triangle \mathrm{RBD} 2$. The large number of peaks in the central area of the spectra of both $\mathrm{hStau} 1{ }^{55}$ _FL and hStau $1{ }^{55}$ _ $\triangle \mathrm{RBD} 2$ indicates the presence of a large number of amino acids in disordered regions, this agrees well with the presence of long unstructured linkers that confer flexibility on the proteins. Moreover, the similar lineshapes and lack of significant chemical shift perturbations between the spectra recorded for individual and tandem domains (Supplemental Figs. S4, S5) show 

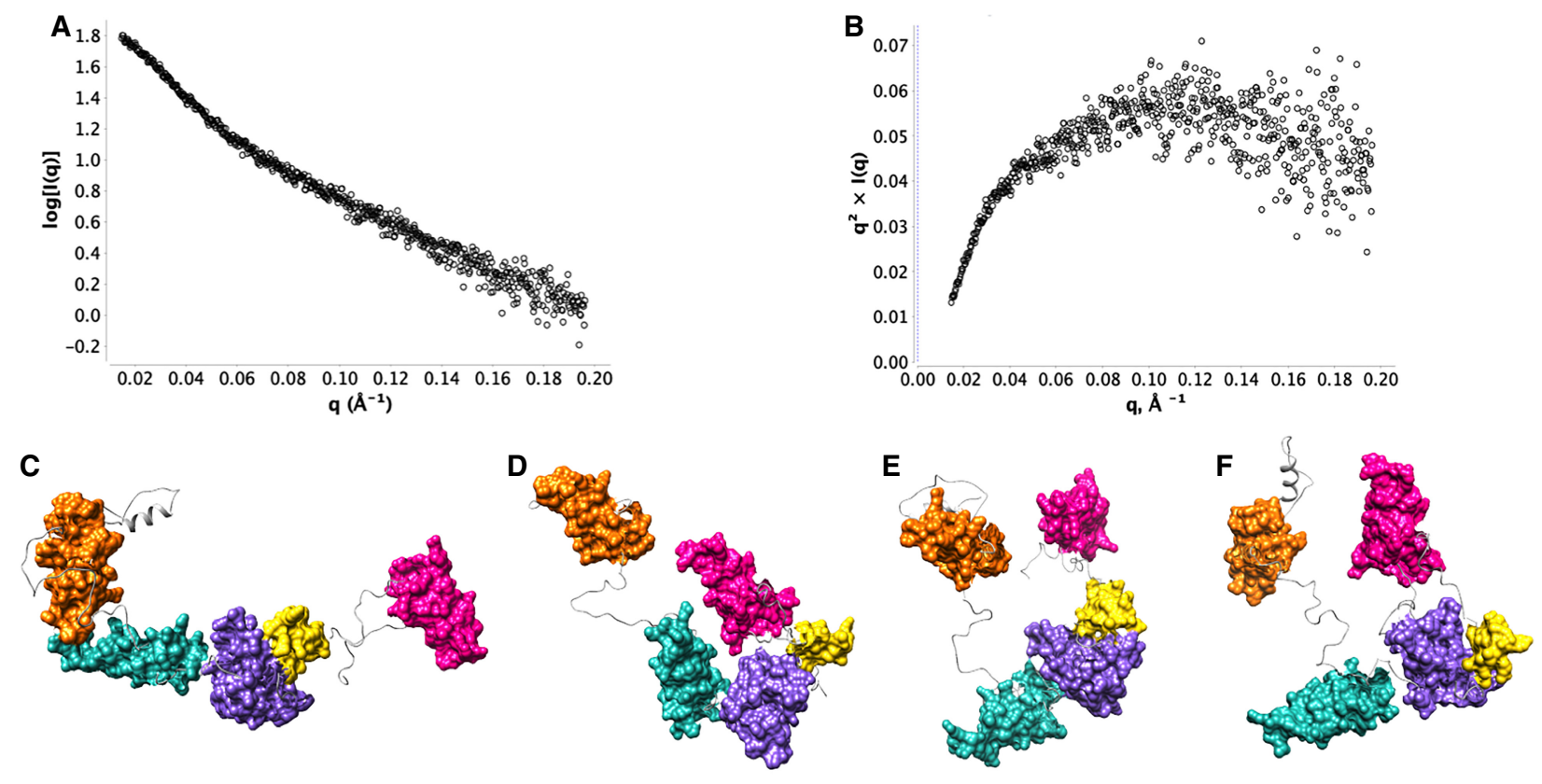

Domains: RBD3, RBD4, TBD, SSM, RBD5.

FIGURE 2. Small-angle X-ray scattering (SAXS) of Staufen proteins. (A) SAXS intensity curve; (B) Kratky analysis; (C-F) EOM models generated for hStau1 ${ }^{55} \_\mathrm{RBD} 2$ at $60 \mu \mathrm{M}\left[R_{\mathrm{g}}\right.$ ensemble $=50.26 \AA, D_{\max }$ ensemble $\left.=166.96 \AA, R_{\text {sigma }}=\sim 80.2 \%(\sim 85.68 \%)\right]$.
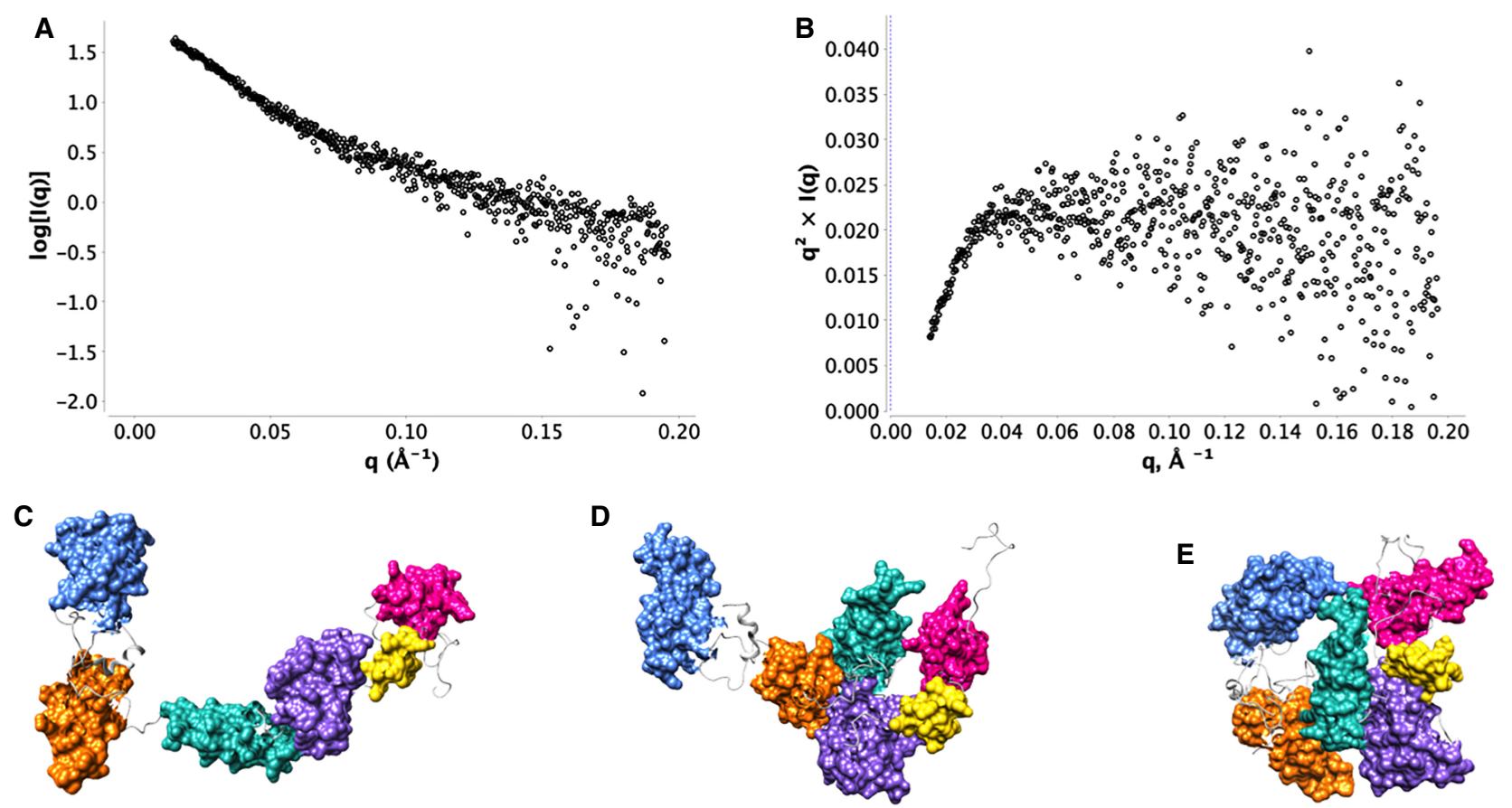

Domains: RBD2, RBD3, RBD4, TBD, SSM, RBD5.

FIGURE 3. Small-angle X-ray scattering (SAXS) of Staufen proteins. (A) SAXS intensity curve; (B) Kratky analysis; (C-E) EOM models generated for $\mathrm{hStau} 1^{55}{ }_{\mathrm{F}} \mathrm{FL}$ at $40 \mu \mathrm{M}\left[R_{\mathrm{g}}\right.$ ensemble $=48.11 \AA$, $D_{\max }$ ensemble $\left.=155.2 \AA, R_{\text {sigma }}=\sim 74.45 \%(\sim 86.17 \%)\right]$. 

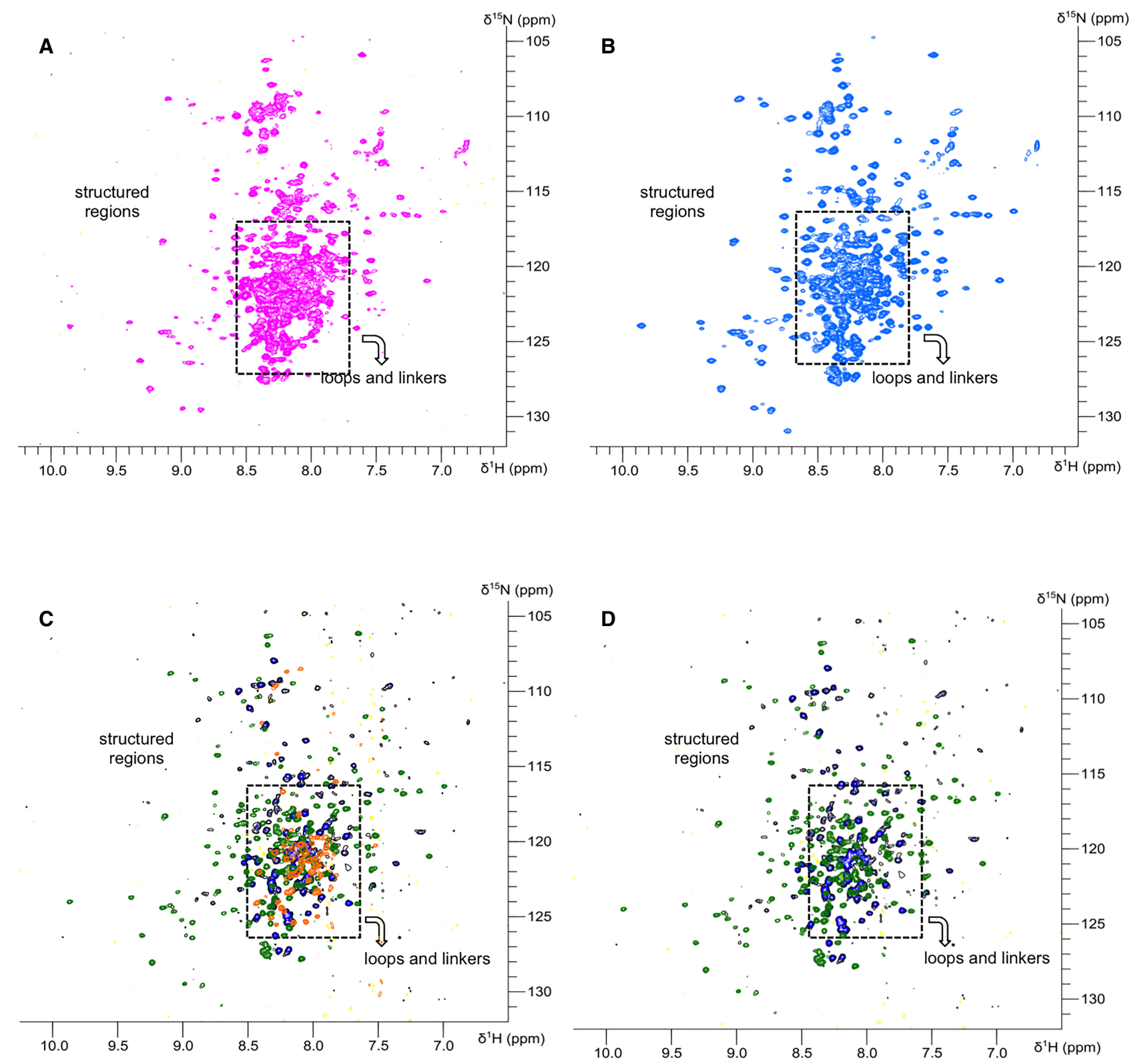

FIGURE 4. NMR analysis of Staufen proteins. (A) NMR spectra of hStau1 ${ }^{55}$ _FL. (B) NMR spectra of hStau1 ${ }^{55}$ _DRBD2. (C) Reconstitution of hStau ${ }^{55}$ _FL spectra by overlapping of RBD2, RBD3-RBD4, and TBD-SSM/RBD5 NMR spectra. (D) Reconstitution of hStau $1^{55}$ _DRBD2 spectra by overlapping of RBD2, RBD3-RBD4, and TBD-SSM/RBD5 NMR spectra.

that any interactions between adjacent domains are very limited and that each of the domains constituting the tandem constructs tumbles independently in solution, thanks to the intervening linkers flexibility. In addition, it is possible to reconstitute the spectra for hStau1 ${ }^{55}$ _ FL and $h S \operatorname{tau} 1{ }^{55} \_$RBD2 almost entirely by overlaying those obtained for individual domains, showing that the domains tumble independently in the full-length protein.

Together, our data suggest that hStau $1^{55}$ is an extremely flexible protein and its domains can adopt several positions relative to each other, without interdomain interactions. Thanks to the flexibility of the linkers, the protein adopts an elongated conformation in solution and its domains behave as beads on a string. Connector regions are crucial players in Staufen allostery and conformational changes, in line with recent studies on the role of the dynamic linker in the modulation of protein function (Papaleo et al. 2016).

\section{Linker flexibility mediates RBD3 and RBD4 rearrangement}

The fitting of a representative subset of SAXS models for individual or tandem domains in the SAXS models of 
hStau ${ }^{55} \_\Delta$ RBD2 and hStau $1^{55}$ FL is shown in Figures 5, 6, respectively. The SAXS data and models obtained for individual and tandem domains are shown in Supplemental Figures S6-S14. A representative subset of solution scattering models of individual and tandem domains (Fig. 5B) was chosen in order to interpret the domains rearrangement observed in the models corresponding to the different conformations that hStau ${ }^{55} \_\Delta \mathrm{RBD} 2$ adopts in solution (Fig. 5A). From the fitting proposed in Figure $5 \mathrm{C}$, it is possible to observe that in hStau $1^{55} \_\mathrm{RBD} 2$ the linker connecting RBD3 to RBD4 can be completely or partially distended. SAXS models obtained for the construct RBD3-RBD4 well describe the behavior of these two domains also when they belong to the truncated protein, showing that the presence of TBD and SSM/ RBD5 does not have a great impact on RBD3-RBD4 rearrangements. On the contrary, the models obtained for the construct RBD3-RBD4 cannot be used for the interpretation of domains rearrangements in $\mathrm{hStau} 1^{55}$ _ $\mathrm{FL}$
(Fig. 6A) and a different subset of solution scattering models (Fig. 6B) needs to be used in the fitting to describe the conformational changes of the full-length protein (Fig. 6C). In fact, the solution models obtained for the tandem domain RBD3-RBD4 (Supplemental Fig. S11) show that a long linker, which is extremely elongated, connects these two domains. On the other hand, our hStau $1^{55}$ FL models show the coexistence of three main conformations in the solution for which it is interesting to notice the relative movement of RBD3 and RBD4 and their closer proximity, possibly in order to elicit the binding of RNA targets (Fig. 7). In the more elongated model of the full-length protein (model 1), RBD3 and RBD4 are in an "open" conformation that resembles the one assumed by the tandem domain on its own. However, in the other two models (2 and 3), RBD3 and RBD4 are "pulled" toward each other by conformational changes of the connecting linker, interestingly resembling the recently deposited structure of the hStau1 ${ }^{63}$ RBD3-RBD4
A
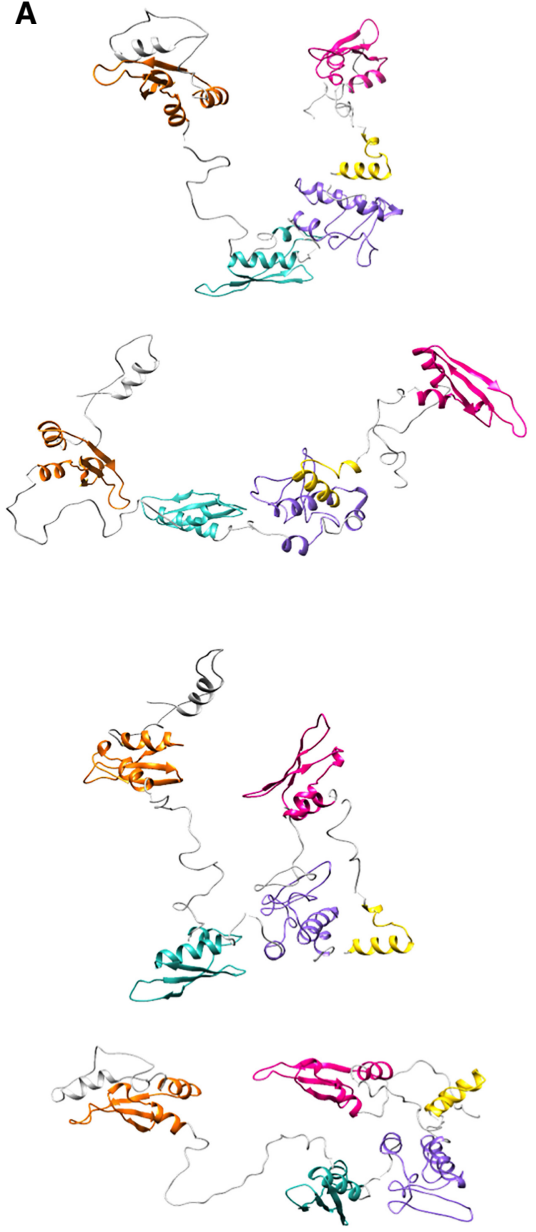

B
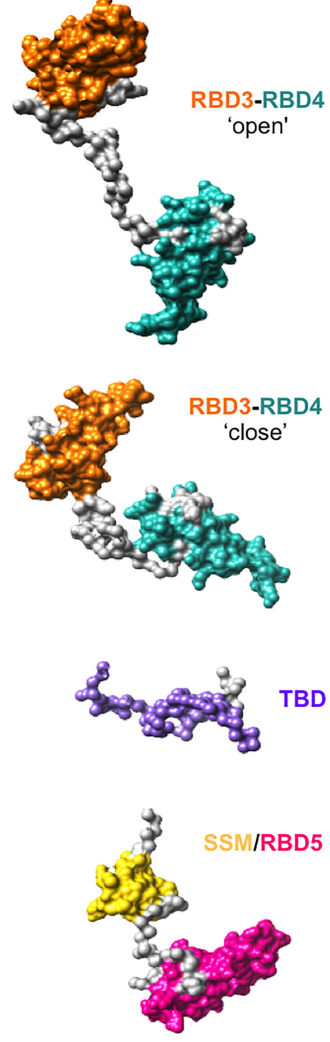

C
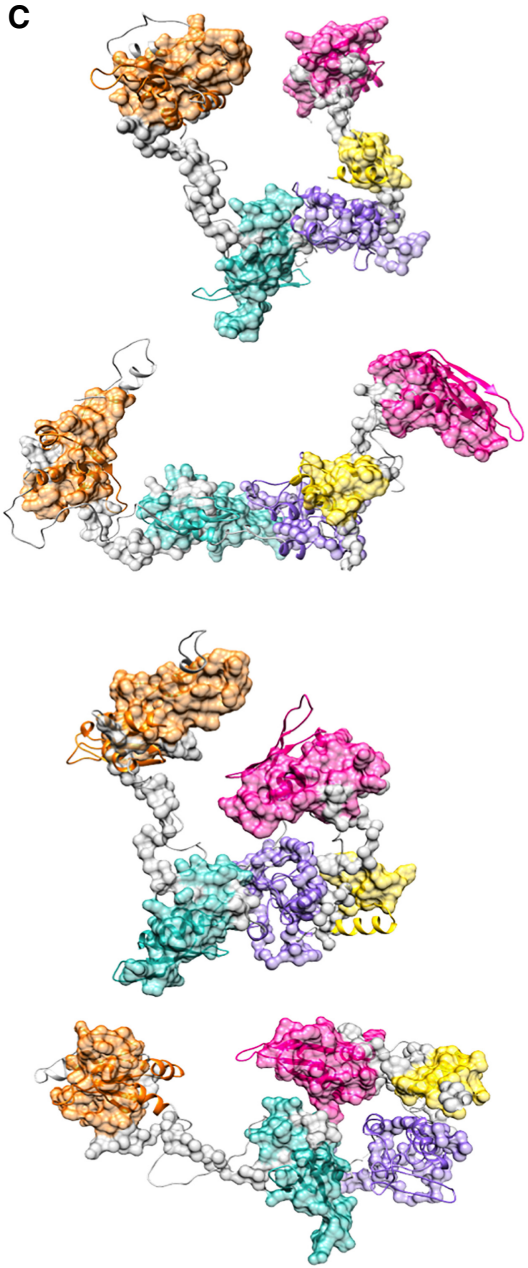

FIGURE 5. Fitting of SAXS models for Staufen 1 domains in the SAXS models obtained for hStau1 ${ }^{55}$ _DRBD2. (A) EOM models generated for hStau $1{ }^{55}$ _DRBD2. (B) Subset of selected domains and tandem domains models. (C) Fitting of representative models for domains and tandem domains in the EOM models generated for hStau1 ${ }^{55}{ }_{-}$DRBD2. 
A
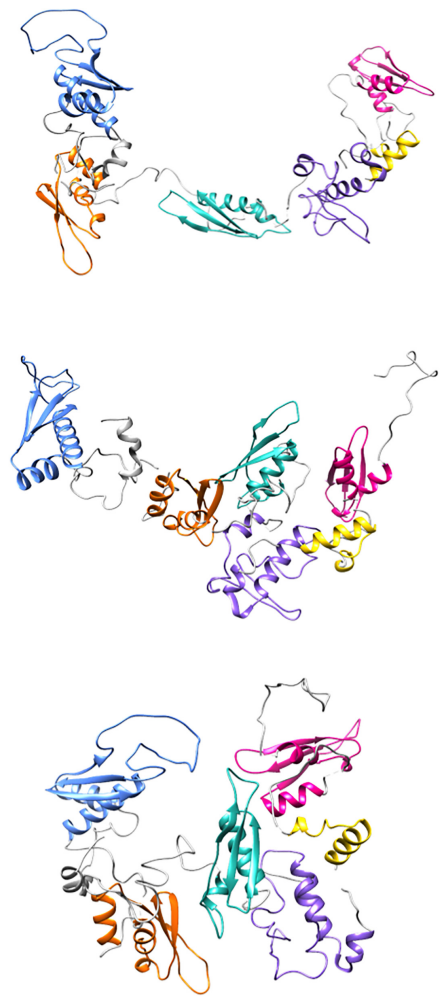

B
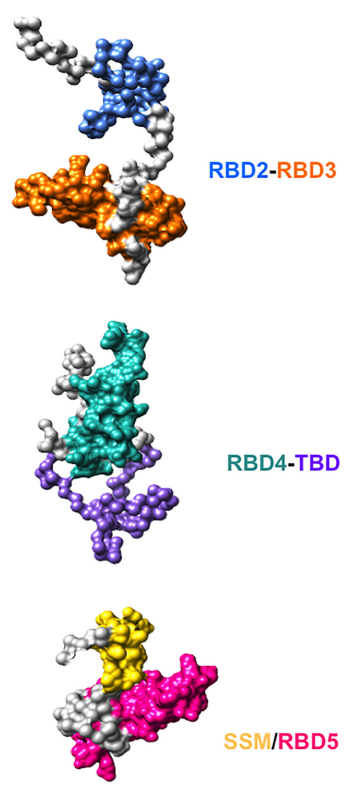
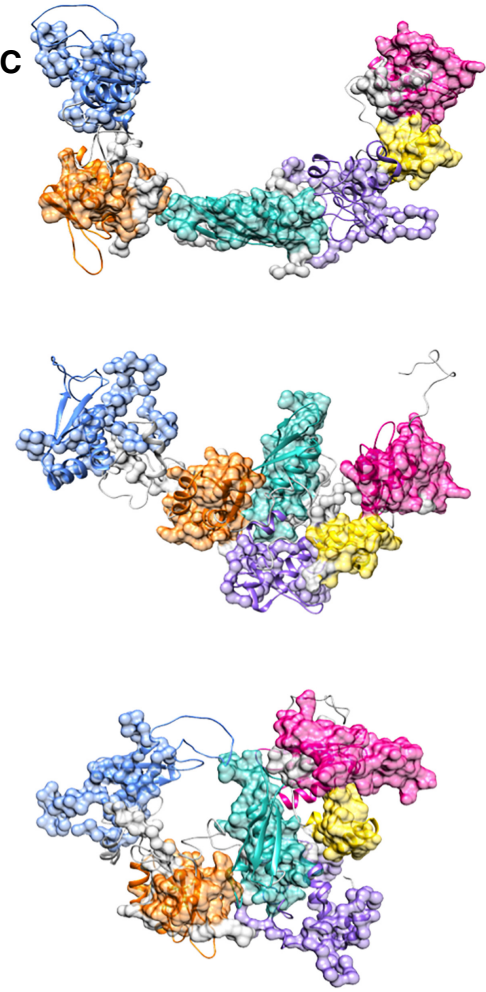

FIGURE 6. Fitting of SAXS models for Staufen 1 domains in the SAXS models obtained for hStau1 ${ }^{55}$ _FL. (A) EOM models generated for hStau ${ }^{55}$ _FL. (B) Subset of selected domains and tandem domains models. (C) Fitting of representative models for domains and tandem domains in the EOM models generated for hStau $1^{55}$ FL.

construct bound to dsRNA (Lazzaretti et al. 2018) (represented in red and blue in Fig. 7).

Taken together, these data show that not only the presence of RBD2 triggers a spatial reorganization of RBD3 and RBD4, which is indeed mediated by the linker between these last two domains, but also the importance of RBD3-RBD4 relative position and rearrangements on dsRNA binding. Therefore, we propose that these two domains can change mutual orientation depending on the structure of the RNA target, in order to effectively bind different substrates in distinct biological contexts. Moreover, RBDs that are not involved in the binding of RNAs, such as RBD2 and RBD5, can adopt multiple conformations in the full-length protein, not only to elicit protein dimerization, but also to regulate hStau1 structural plasticity and multifunctionality in vivo. All in all, our solution studies demonstrate that Stau protein can adopt several conformations thanks to long linkers that facilitate domains rearrangements, providing a clue on the structural background for the role of Stau in multiple biological pathways. This would be reminiscent of the remodeling of flexible components in the presence of target nucleic acid, which has been seen in other DNA and RNA binding proteins (Tsutakawa et al. 2014) that can change overall shape by altering their protein conformations to switch among their multiple functions.

\section{Conclusions}

Canonical RBDs are composed of an $\alpha-\beta-\beta-\beta-\alpha$ secondary structure that folds in three dimensions to recognize dsRNA. Recently, structural and functional studies of divergent $\mathrm{RBDs}$ revealed adaptations that include intra- and/or intermolecular protein interactions, sometimes in the absence of detectable dsRNA-binding ability (Krovat and Jantsch 1996; Gleghorn and Maquat 2014). The number of canonical RBDs per polypeptide is highly variable in RBD-containing proteins, ranging from one to five (Macrae et al. 2006; Barraud and Allain 2012; Thomas and Beal 2017). There is no clear correlation between the number of RBDs and dsRNA-binding affinity. Moreover, the extent of the contribution of specific RBDs to RNA substrate specificity is still an open question. Structural information reported on dsRBPs carrying one or more canonical RBD, such as Dicer (Macrae et al. 2006) and ADARs (Barraud and Allain 2012; Thomas and Beal 2017) highlights the importance of neighboring domains for substrate specificity and enzymatic activity. Conformational flexibility of Dicer protein is also proposed to play a central role in dsRNA recognition and processing (Macrae et al. 2006) and this could be extended to other RBD-containing proteins, such as hStau1. The lack of structural information on this protein, for which to date we only had three- 


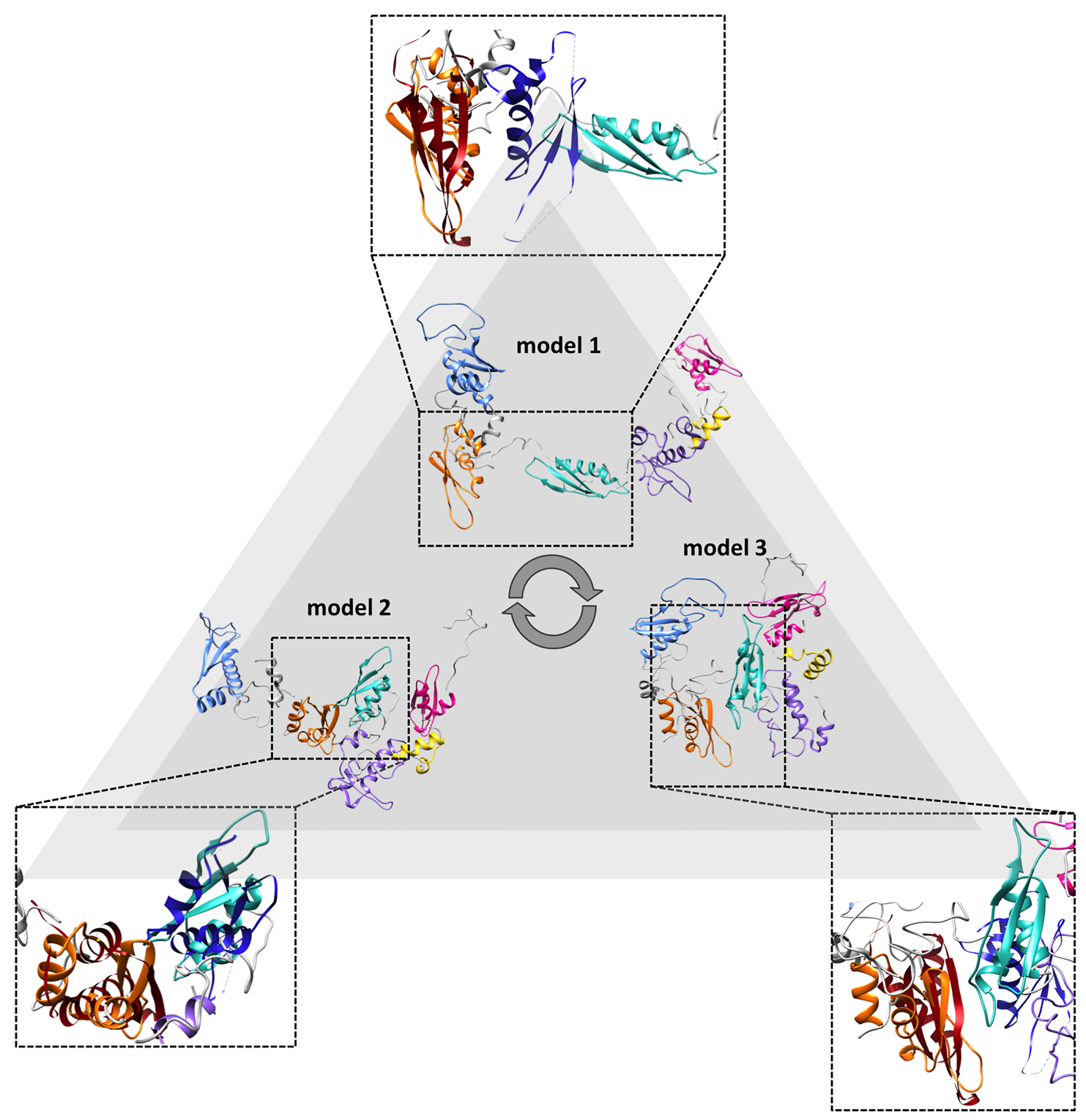

FIGURE 7. RBD3 and RBD4 rearrangements in hStau1 ${ }^{55}$ _FL can explain its plasticity in the binding of diverse dsRNA targets. The SAXS models obtained for hStau1 ${ }^{55}$ FL show that RBD3 (orange) and RBD4 (cyan) transit from an "open" to a "more closed" conformation. A direct comparison of our models with the crystal structure of the hStau1 ${ }^{63}$ RBD3-RBD4 construct bound to Arf1 SBS ${ }^{43}$ (RBD3 displayed in red and RBD 4 in blue) shows that RBD3 and RBD4 are "pulled" toward each other by conformational changes of the connecting linker, possibly in order to elicit dsRNA binding.

dimensional models of truncation mutants, in isolation or in complex with short RNA sequences or truncated protein interactors (Ramos et al. 1999, 2000; Gleghorn et al. 2013; Gleghorn and Maquat 2014; Jia et al. 2015; Lazzaretti et al. 2018) made its functional understanding particularly challenging. Here, we provided for the first time structural information on the full-length hStau1 protein, using an integrated structural biology approach. Combination of hydrodynamic Materials and Materials and Methods, homology modeling, SAXS and NMR allowed us to show that Stau1 is a highly flexible protein, that recoils in solution from an elongated to a compact conformation in which the domains are in closer proximity but not interacting with each other. In this perspective, we propose that the flexible interdomain loops possess a regulatory role in hStau1 activity, allowing a high degree of freedom for recognition and binding of diverse RNA and protein targets and for the subsequent involvement of hStau1 in very diverse aspects of RNA metabolism and regulation. Interestingly, post-translational modifications have been identified in loops and linkers regions within hStau1 protein (Rigbolt et al. 2011; Zhou et al. 2013; Guo et al. 2014), which could be involved in the regulation of domains rearrangement or protein-protein interactions. 
As far as the dimerization of hStau1 is concerned, we show that this is dependent on the presence of RBD2 and on protein concentration. Consistently with what has been shown crystallographically, the SSM-RBD5 construct dimerized in solution (Supplemental Fig. S2). SAXS experiments show that the full-length protein adopts at least three main conformations in solution, which therefore can explain its ability to bind diverse RNA targets and protein partners. Our data provide the first structural insight into the "Swiss knife" mechanism adopted by the Stau $1{ }^{55}$ protein to elicit sometimes contrasting biological functions.

\section{MATERIALS AND METHODS}

\section{Cloning of individual and tandem domains for NMR analysis}

Individual and tandem hStau $1^{55}$ domains were amplified by PCR from pRSET-B-Stau $1^{55}$ vector (Kim et al. 2005) as described in Supplemental Table S1. Purified PCR products and pET28a were digested with $\mathrm{Ndel}$ and HindIIIHF (NEB) for $3 \mathrm{~h}$ at $37^{\circ} \mathrm{C}$. Digested vector was purified from $1 \%$ agarose gel run in TBE using the MinElute Gel Extraction Kit (QIAGEN), whereas digested inserts were purified using the QIAquick PCR Purification Kit (QIAGEN). Ligations between the vector and the individual inserts (in ratio 1:3) were performed using the Quick Ligation Kit (NEB) for $5 \mathrm{~min}$ at room temperature (RT). Escherichia coli XL1-Blue cells were transformed by the heat-shock method with $2.5 \mu \mathrm{L}$ of the ligation reactions and plated in LB agar plates containing $50 \mu \mathrm{g} / \mathrm{mL}$ kanamycin. After overnight (o/n), for each of the transformations, $15 \mathrm{~mL}$ of LB supplemented with $50 \mu \mathrm{g} / \mathrm{mL}$ kanamycin were inoculated with a single colony and the cultures were grown at $37^{\circ} \mathrm{C}$ $\mathrm{o} / \mathrm{n}$. Subsequently, plasmid DNA was purified using the Wizard Plus SV Minipreps DNA Purification Systems and sequenced.

\section{Protein overexpression and purification for SAXS, $A U C$, and EM experiments}

Recombinant proteins were overexpressed in E. coli Rosetta pLysS cells transformed by heat-shock with pRSET-B vectors containing either hStau155_FL (Kim et al. 2005), hStau 1 ${ }^{55}{ }_{-} \mathrm{RBD} 2$ or pET28a containing individual domains (RBD2, RBD3, RBD4, TBD, $\mathrm{SSM} / \mathrm{RBD} 5$ ) or tandem domains (RBD2-RBD3, RBD3-RBD4, RBD4-TBD, TBD-SSM/RBD5). Starting cultures were grown in LB medium containing $50 \mu \mathrm{g} / \mathrm{mL}$ ampicillin (for hStau $1^{55}$ FL and hStau1 ${ }^{55} \_$RBD2) or $50 \mu \mathrm{g} / \mathrm{mL}$ kanamycin (for individual and tandem domains) and $34 \mu \mathrm{g} / \mathrm{mL}$ chloramphenicol at $37^{\circ} \mathrm{C}$ $\mathrm{o} / \mathrm{n}$. One milliliter of overnight culture was inoculated in $1 \mathrm{~L}$ of terrific broth (TB) medium supplemented with antibiotics and cells were grown to an $0.6 \mathrm{OD}_{600}$. Protein overexpression was induced by adding $0.5 \mathrm{mM}$ IPTG and culturing the cells at $27^{\circ} \mathrm{C}$ overnight. Cells were harvested by centrifugation at $5000 \mathrm{rpm}$ on a Beckman Avanti J-20 XP centrifuge with JLA 8.1000 rotor for $20 \mathrm{~min}$ and washed once with phosphate buffered saline (PBS) solution; cell pellets were aliquoted and stocked at $-80^{\circ} \mathrm{C}$. Frozen aliquots were thawed and lysed by sonication in $20 \mathrm{~mL}$ lysis buffer (25 mM HEPES $\mathrm{pH}$ 7.5, $1 \mathrm{M} \mathrm{GndCl,} 20 \mathrm{mM}$ imidazole, 1\%
Triton X-100, $400 \mu \mathrm{L}$ Complete EDTA-free protease inhibitors [50X] and $2 \mu \mathrm{L}$ benzonase) followed by $30 \mathrm{~min}$ of incubation on ice. Soluble protein extracts were separated from cell pellets by centrifugation at 15,000 rpm for $30 \mathrm{~min}$. 6-His tagged hStau 1 proteins were purified by nickel chromatography using HisTrap FF columns (GE Healthcare) equilibrated in washing buffer $(25 \mathrm{mM}$ HEPES pH 7.5, $1 \mathrm{M} \mathrm{GndCl,} 20 \mathrm{mM}$ imidazole). Elution was performed with $20 \mathrm{mM}-1 \mathrm{M}$ imidazole gradient. Fractions containing the protein of interest were pooled and concentrated to $10 \mathrm{mg} /$ $\mathrm{mL}$ prior to size exclusion chromatography on Superdex200 (hStau $1^{55}$ FL and $\mathrm{hStau} 1^{55} \_\Delta \mathrm{RBD} 2$ ) or Superdex 75 (individual and tandem domains) gel filtration columns (GE Healthcare) in buffer A (25 mM HEPES pH 7.5, 100 mM KCl, 10 mM MgCl2, $200 \mathrm{mM}$ L-Arg $\mathrm{HCl}$ ). Overexpression and purification efficiency were monitored by SDS-PAGE analysis on 12\%, 15\%, and $18 \%$ gels stained with SimpleBlue (Life Technologies). Fractions were also analyzed by western blot; the nitrocellulose membrane was incubated o/n at $4^{\circ} \mathrm{C}$ with anti-His antibody conjugated with alkaline phosphatase $(1: 4000)$ and developed using the SIGMAFAST BCIP/NBT reagent. Size exclusion chromatography and SDS-PAGE analysis for hStau $1^{55}$ _FL and hStau $1{ }^{55}{ }_{-} \mathrm{RBD} 2$ are shown in Supplemental Figure S1, those for individual and tandem domains are shown in Supplemental Figure S2.

\section{Protein overexpression and purification for NMR analysis}

E. coli Rosetta pLysS cells were transformed by heat-shock with pET-28 vectors carrying inserts for hStau $1^{55}$ FL, hStau $1^{55} \_$RBD2, individual domains (RBD2, RBD3, RBD4, TBD, $\mathrm{SSM} / \mathrm{RBD} 5$ ), and tandem domains (RBD2-RBD3, RBD3-RBD4, RBD4-TBD, TBD-SSM/RBD5). Starting cultures were grown as described above and the following $d$ cells were cultured to OD600 $=2$ in TB medium supplemented with antibiotics. Cells were harvested by centrifugation at $5000 \mathrm{rpm}$ on a Beckman AvantiTM J-20 XP centrifuge with JLA 8.1000 rotor for 20 min, resuspended in $\mathrm{M} 9$ minimal medium $(1 \times \mathrm{M} 9,2 \mathrm{mM} \mathrm{MgSO}, 0.1$ $\mathrm{mM} \mathrm{CaCl} 2$ ) and grown for $30 \mathrm{~min}$ at $37^{\circ} \mathrm{C}$. Successively, the minimal medium was supplemented with $400 \mu \mathrm{L}$ thiamine [50 mg/ $\mathrm{mL}$ ], filter-sterilized glucose [3 $\mathrm{g} / \mathrm{L}$ ] and filter-sterilized ISOGRO$15 \mathrm{~N}$ Powder-Growth Medium [1 g/L]. Protein overexpression was induced by adding $0.5 \mathrm{mM}$ IPTG and culturing the cells at $27^{\circ} \mathrm{C}$ overnight. Purification of Staufen proteins was performed as described above, but replacing HEPES with $20 \mathrm{mM}$ potassium phosphate buffer $\mathrm{pH} 7.6$ in all buffers used. To prevent the formation of disulfide bonds, $2 \mathrm{mM}$ Tris(2-carboxyethyl)phosphine hydrochloride (TCEP) was added to the purified proteins.

\section{Analytical ultracentrifugation (AUC)}

The experiments were performed at 40,000 rpm, using a Beckman XL-I analytical ultracentrifuge equipped with an An50Ti rotor. Data were recorded using both absorbance (at 280 and $260 \mathrm{~nm}$ ) and interference optical detection systems. The density and viscosity of the buffer were measured experimentally using a DMA 5000M densitometer equipped with a Lovis 200ME viscometer module. The partial specific volume for the protein was calculated using SEDNTERP from the amino acid sequence. 
Data were processed using SEDFIT, fitting to the $\mathrm{c}(\mathrm{s})$ model. Figures were made using GUSSI (Lebowitz et al. 2002).

\section{SEC-MALS}

SEC-MALS experiments were performed using a Superdex 200 $10 / 300$ Increase column (GE Healthcare) connected to an AktaPure 25 System (GE Healthcare). The protein sample (100 $\mu \mathrm{L}$ ) was loaded onto the gel filtration column and eluted with one column volume $(24 \mathrm{~mL})$ of buffer $A$, at a flow rate of 0.7 $\mathrm{mL} / \mathrm{min}$. The eluting protein was monitored using a DAWN HELEOS-II 18-angle light scattering detector (Wyatt Technologies) equipped with a WyattQELS dynamic light scattering module, a U9-M UV/Vis detector (GE Healthcare), and an Optilab T-rEX refractive index monitor (Wyatt Technologies). Data were analyzed by using the Astra software (Wyatt Technologies) using a refractive increment value of $0.185 \mathrm{~mL} / \mathrm{g}$.

\section{Small angle X-ray scattering and modeling}

SAXS data for $h S t a u 1^{55}$ _FL and $h S t a u 1^{55} \_$RBD2 were collected at B21, Diamond Light Source (Harwell, UK). Fifty-five microliters of each protein sample $(\sim 10 \mathrm{mg} / \mathrm{mL})$ were loaded onto a Superdex200 column (GE Healthcare), controlled by an Agilent HPLC system, coupled to an in-vacuum SAXS flow cell. HPLCSAXS traces were processed using ScÅtter. High-resolution structures of individual domains were used as rigid bodies and constraints in the model generation. In our analysis, the modeled structures of the individual domains of Staufen 1 were obtained using the Phyre2 web portal (Kelley et al. 2015). Human Staufen1 RBD3 was modeled by homology based on the NMR structure of $D$. melanogaster Staufen RBD3 (PDB ID: 1EKZ) (Ramos et al. 2000). The structure of human Staufen1 RBD4 was obtained by homology modeling based on the mouse RBD4 (PDB ID: 1UHZ). The structures of SSM and RBD5, were extracted from the structure solved by X-ray crystallography (PDB ID: 4DKK) (Gleghorn et al. 2013) and treated as two separate domains in this analysis, allowing complete interdomain loop flexibility. To obtain a more complete set of structural information to use as constraints for the interpretation of the SAXS data, the sequences of the 6-His + linker + RBD2 domain of $\mathrm{hStau} 1{ }^{55}{ }_{-} \mathrm{FL}$, the 6 -His + linker of hStau $1^{55} \_$RBD2 and the TBD were modeled using the Phyre2 server (Kelley et al. 2015). The program EOM 2.0 (Tria et al. 2015) was used to obtain the models of hStau $1^{55}$ FL protein and deletion mutants. A pool of 10,000 independent models was generated, based on the sequence of $\mathrm{hStau} 1^{55} \mathrm{FL}$, or hStau $1^{55} \_$RBD2, and on constraints, we generated by homology modeling. After the creation of the pool of models, EOM (Tria et al. 2015) runs a genetic algorithm that compares the average theoretical scattering intensity from the ensemble of 10,000 conformations with the experimental scattering data and selects the models that best describe the experimental data, taking into account the constraints used as input (in this case, the homology models of individual domains).

SAXS data for individual domains and tandem domains were collected at B21, Diamond Light Source (Harwell, UK). Fifty-five microliters of each protein sample $(\sim 10 \mathrm{mg} / \mathrm{mL})$ were loaded onto a Superdex75 column (GE Healthcare), controlled by an Agilent HPLC system, coupled to an in-vacuum SAXS flow cell.
HPLC-SAXS traces were processed using ScÅtter. Data were analyzed using different strategies depending on their flexibility level. Models for individual domains RBD3 and RBD4 were obtained using ScÅtter and DAMMIN and the tandem domain TBD_SSM/ RBD5 was modeled using BUNCH (ATSAS). All the other domains, showing higher degree of flexibility, were modeled with EOM, as described above.

\section{NMR spectroscopy}

hStau $1^{55} \_\mathrm{FL}, \mathrm{hStau} 1^{55} \_\mathrm{RBD} 2$, individual and tandem domains were studied by NMR spectroscopy. Thirty microliters of $\mathrm{D}_{2} \mathrm{O}$ were added to $570 \mu \mathrm{L}$ of protein in $20 \mathrm{mM}$ potassium phosphate $\mathrm{pH} 7.5,100 \mathrm{mM} \mathrm{KCl}, 10 \mathrm{mM} \mathrm{MgCl} 2,200 \mathrm{mM}$ L-Arg HCl, 2mM TCEP at a suitable concentration for NMR experiments (Supplemental Table S2). ${ }^{15} \mathrm{~N},{ }^{1} \mathrm{H}$-TROSY-HSQC spectra (Weigelt 1998) were acquired at $298 \mathrm{~K}$ using a Bruker AVANCE IIIHD $600 \mathrm{MHz}$ spectrometer equipped with a $5 \mathrm{~mm} \mathrm{TCl}$ cryoprobe. Data were processed using the Bruker TopSpin software and figures were generated using the CCPN analysis 2.4 software (Vranken et al. 2005).

\section{SUPPLEMENTAL MATERIAL}

Supplemental material is available for this article.

\section{ACKNOWLEDGMENTS}

We are grateful to Lynne Maquat for the kind gift of the pRSET-BStau $1^{55}$ vector (Kim et al. 2005). We acknowledge Eve Hartswood and Amy Whittaker for some initial protein production tests. We are grateful to Drs. Robert Rambo, Nathan Cowieson, Nikul Khunti, and Katsuaki Inoue (Diamond Light Source, UK) for assistance with data collection at B21, Diamond Light Source, Dr. Daniel Myatt (ISIS Pulsed Neutron and Muon Source) for help with the initial scattering experiments, and to Dr. David Scott for access to biophysical instrumentation at the Research Complex at Harwell. S.V. is supported by an ISIS Neutron Source/University of Edinburgh joint PhD Studentship (STFC/ SA15). G.C. was supported by a Darwin Trust of Edinburgh PhD Studentship.

Received July 3, 2019; accepted December 9, 2019.

\section{REFERENCES}

Allison R, Czaplinski K, Git A, Adegbenro E, Stennard F, Houliston E, Standart N. 2004. Two distinct Staufen isoforms in Xenopus are vegetally localized during oogenesis. RNA 10: 1751-1763. doi:10.1261/rna.7450204

Baker KS, Chow EJ, Goodman PJ, Leisenring WM, Dietz AC, Perkins JL, Chow L, Sinaiko A, Moran A, Petryk A, et al. 2013. Impact of treatment exposures on cardiovascular risk and insulin resistance in childhood cancer survivors. Cancer Epidemiol Biomarkers Prev 22: 1954-1963. doi:10.1158/1055-9965.EPI-130610

Banerjee A, Benjamin R, Balakrishnan K, Ghosh P, Banerjee S. 2014. Human protein Staufen-2 promotes HIV-1 proliferation by 
positively regulating RNA export activity of viral protein Rev. Retrovirology 11: 18. doi:10.1186/1742-4690-11-18

Barraud P, Allain FH-T. 2012. ADAR proteins: double-stranded RNA and Z-DNA binding domains. Curr Top Microbiol Immunol 353: 35-60. doi:10.1007/82_2011_145

Bélanger G, Stocksley MA, Vandromme M, Schaeffer L, Furic L, DesGroseillers L, Jasmin BJ. 2003. Localization of the RNA-binding proteins Staufen 1 and Staufen 2 at the mammalian neuromuscular junction. J Neurochem 86: 669-677. doi:10.1046/j.14714159.2003.01883.x

Blackham SL, McGarvey MJ. 2013. A host cell RNA-binding protein, Staufen 1, has a role in hepatitis $C$ virus replication before virus assembly. J Gen Virol 94: 2429-2436. doi:10.1099/vir.0 .051383-0

Bonnet-Magnaval F, Philippe C, Van Den Berghe L, Prats H, Touriol C, Lacazette E. 2016. Hypoxia and ER stress promote Staufen1 expression through an alternative translation mechanism. Biochem Biophys Res Commun 479: 365-371. doi:10.1016/j.bbrc.2016 .09 .082

Boulay K, Ghram M, Viranaicken W, Trépanier V, Mollet S, Fréchina C, DesGroseillers L. 2014. Cell cycle-dependent regulation of the RNA-binding protein Staufen1. Nucleic Acids Res 42: 78677883. doi:10.1093/nar/gku506

Buchner G, Bassi MT, Andolfi G, Ballabio A, Franco B. 1999. Identification of a novel homolog of the Drosophila staufen protein in the chromosome 8q13-q21.1 region. Genomics 62: 113-118. doi:10.1006/geno.1999.6015

Bycroft M, Grünert S, Murzin AG, Proctor M, St Johnston D. 1995 NMR solution structure of a dsRNA binding domain from Drosophila staufen protein reveals homology with the $\mathrm{N}$-terminal domain of ribosomal protein S5. EMBO J 14: 3563-3571. doi:10 $.1002 / j .1460-2075.1995 . t b 07362 . x$

Chatel-Chaix L, Clement J-F, Martel C, Beriault V, Gatignol A, DesGroseillers L, Mouland AJ. 2004. Identification of Staufen in the human immunodeficiency virus type 1 Gag ribonucleoprotein complex and a role in generating infectious viral particles. Mol Cell Biol 24: 2637-2648. doi:10.1128/MCB.24.7.2637-2648.2004

Chatel-Chaix L, Boulay K, Mouland AJ, Desgroseillers L. 2008. The host protein Staufen 1 interacts with the Pr55Gag zinc fingers and regulates HIV-1 assembly via its $\mathrm{N}$-terminus. Retrovirology 5: 41. doi:10.1186/1742-4690-5-41

Cho H, Kim KM, Han S, Choe J, Park SG, Choi SS, Kim YK. 2012. Staufen1-mediated mRNA decay functions in adipogenesis. Mol Cell 46: 495-506. doi:10.1016/j.molcel.2012.03.009

de Lucas S, Peredo J, Marión RM, Sanchez C, Ortin J. 2010. Human Staufen 1 protein interacts with influenza virus ribonucleoproteins and is required for efficient virus multiplication. J Virol 84: 76037612. doi:10.1128/JVI.00504-10

de Lucas S, Oliveros JC, Chagoyen M, Ortín J. 2014. Functional signature for the recognition of specific target mRNAs by human Staufen1 protein. Nucleic Acids Res 42: 4516-4526. doi:10 .1093/nar/gku073

Dixit U, Pandey AK, Mishra P, Sengupta A, Pandey VN. 2016. Staufen1 promotes HCV replication by inhibiting protein kinase $\mathrm{R}$ and transporting viral RNA to the site of translation and replication in the cells. Nucleic Acids Res 44: 5271-5287. doi:10.1093/nar/gkw312

Duchaine TF, Hemraj I, Furic L, Deitinghoff A, Kiebler MA, DesGroseillers L. 2002. Staufen2 isoforms localize to the somatodendritic domain of neurons and interact with different organelles. J Cell Sci 115: 3285-3295.

Fernández Moya SM, Kiebler MA. 2015. CLIPing Staufen to secondary RNA structures: size and location matter! Bioessays 37: 10621066. doi:10.1002/bies. 201500052

Furic L, Maher-Laporte M, DesGroseillers L. 2008. A genome-wide approach identifies distinct but overlapping subsets of cellular
mRNAs associated with Staufen1- and Staufen2-containing ribonucleoprotein complexes. RNA 14: 324-335. doi:10.1261/rna .720308

Gautrey H, McConnell J, Hall J, Hesketh J. 2005. Polarised distribution of the RNA-binding protein Staufen in differentiated intestinal epithelial cells. FEBS Lett 579: 2226-2230. doi:10.1016/j.febslet 2005.02.074

Gautrey H, McConnell J, Lako M, Hall J, Hesketh J. 2008. Staufen1 is expressed in preimplantation mouse embryos and is required for embryonic stem cell differentiation. Biochim Biophys Acta 1783: 1935-1942. doi:10.1016/j.bbamcr.2008.05.017

Gleghorn ML, Maquat LE. 2014. 'Black sheep' that don't leave the double-stranded RNA-binding domain fold. Trends Biochem Sci 39: 328-340. doi:10.1016/j.tibs.2014.05.003

Gleghorn ML, Gong C, Kielkopf CL, Maquat LE. 2013. Staufen1 dimerizes through a conserved motif and a degenerate dsRNA-binding domain to promote mRNA decay. Nat Struct Mol Biol 20: 515524. doi: $10.1038 / \mathrm{nsmb} .2528$

Gong C, Maquat LE. 2011. IncRNAs transactivate STAU1-mediated mRNA decay by duplexing with 3' UTRs via Alu elements. Nature 470: 284-288. doi:10.1038/nature09701

Gong C, Kim YK, Woeller CF, Tang Y, Maquat LE. 2009. SMD and NMD are competitive pathways that contribute to myogenesis: effects on PAX3 and myogenin mRNAs. Genes Dev 23: 54-66. doi:10.1101/gad.1717309

Guo A, Gu H, Zhou J, Mulhern D, Wang Y, Lee KA, Yang V, Aguiar M, Kornhauser J, Jia X, et al. 2014. Immunoaffinity enrichment and mass spectrometry analysis of protein methylation. Mol Cell Proteom 13: 372-387. doi:10.1074/mcp.0113.027870

Jia M, Shan Z, Yang Y, Liu C, Li J, Luo Z-G, Zhang M, Cai Y, Wen W, Wang W. 2015. The structural basis of Miranda-mediated Staufen localization during Drosophila neuroblast asymmetric division. Nat Commun 6: 8381. doi:10.1038/ncomms9381

Kelley LA, Mezulis S, Yates CM, Wass MN, Sternberg MJ. 2015. The Phyre2 web portal for protein modeling, prediction and analysis. Nat Protoc 10: 845-858. doi:10.1038/nprot.2015.053

Kim YK, Furic L, Desgroseillers L, Maquat LE. 2005. Mammalian Staufen1 recruits Upf1 to specific mRNA $3^{\prime}$ UTRs so as to elicit mRNA decay. Cell 120: 195-208. doi:10.1016/j.cell.2004 .11 .050

Kim MY, Park J, Lee JJ, Ha DH, Kim J, Kim CG, Hwang J, Kim CG. 2014. Staufen1-mediated mRNA decay induces Requiem mRNA decay through binding of Staufen1 to the Requiem $3^{\prime} U T R$. Nucleic Acids Res 42: 6999-7011. doi:10.1093/nar/gku388

Kretz M. 2013. TINCR, staufen1, and cellular differentiation. RNA Biol 10: 1597-1601. doi:10.4161/rna.26249

Krovat BC, Jantsch MF. 1996. Comparative mutational analysis of the double-stranded RNA binding domains of Xenopus laevis RNAbinding protein A. J Biol Chem 271: 28112-28119. doi:10.1074/ jbc.271.45.28112

Laver JD, Li X, Ancevicius K, Westwood JT, Smibert CA, Morris QD, Lipshitz HD. 2013. Genome-wide analysis of Staufen-associated mRNAs identifies secondary structures that confer target specificity. Nucleic Acids Res 41: 9438-9460. doi:10.1093/nar/gkt702

Lazzaretti D, Bandholz-Cajamarca L, Emmerich C, Schaaf K, Basquin C, Irion U, Bono F. 2018. The crystal structure of Staufen 1 in complex with a physiological RNA sheds light on substrate selectivity. Life Sci Alliance 1: e201800187. doi:10.26508/ Isa.201800187

Lebeau G, Maher-Laporte M, Topolnik L, Laurent CE, Sossin W, Desgroseillers L, Lacaille J-C. 2008. Staufen1 regulation of protein synthesis-dependent long-term potentiation and synaptic function in hippocampal pyramidal cells. Mol Cell Biol 28: 28962907. doi:10.1128/MCB.01844-07 
Lebowitz J, Lewis MS, Schuck P. 2002. Modern analytical ultracentrifugation in protein science: a tutorial review. Protein Sci 11: 2067-2079. doi:10.1110/ps.0207702

LeGendre JB, Campbell ZT, Kroll-Conner P, Anderson P, Kimble J, Wickens M. 2013. RNA targets and specificity of Staufen, a double-stranded RNA-binding protein in Caenorhabditis elegans. J Biol Chem 288: 2532-2545. doi:10.1074/jbc.M112.397349

Luo M, Duchaîne TF, DesGroseillers L. 2002. Molecular mapping of the determinants involved in human Staufen-ribosome association. Biochem J 365: 817-824. doi:10.1042/bj20020263

Macrae IJ, Li F, Zhou K, Cande WZ, Doudna JA. 2006. Structure of Dicer and mechanistic implications for RNAi. Cold Spring Harb Symp Quant Biol 71: 73-80. doi:10.1101/sqb.2006.71.042

Marión RM, Fortes P, Beloso A, Dotti C, Ortín J. 1999. A human sequence homolog of Staufen is an RNA-binding protein that is associated with polysomes and localizes to the rough endoplasmic reticulum. Mol Cell Biol 19: 2212-2219. doi:10.1128/MCB.19.3 .2212

Martel C, Macchi P, Furic L, Kiebler MA, Desgroseillers L. 2006. Staufen 1 is imported into the nucleolus via a bipartite nuclear localization signal and several modulatory determinants. Biochem J 393: 245-254. doi:10.1042/BJ20050694

Martel C, Dugre-Brisson S, Boulay K, Breton B, Lapointe G, Armando S, Trepanier V, Duchaine T, Bouvier M, Desgroseillers L. 2010. Multimerization of Staufen1 in live cells. RNA 16: 585-597. doi:10.1261/rna.1664210

Mouland AJ, Mercier J, Luo M, Bernier L, DesGroseillers L, Cohen EA. 2000. The double-stranded RNA-binding protein Staufen is incorporated in human immunodeficiency virus type 1: evidence for a role in genomic RNA encapsidation. J Virol 74: 5441-5451. doi:10.1128/JVI.74.12.5441-5451.2000

Papaleo E, Saladino G, Lambrughi M, Lindorff-Larsen K, Gervasio FL, Nussinov R. 2016. The role of protein loops and linkers in conformational dynamics and allostery. Chem Rev 116: 6391-6423. doi:10.1021/acs.chemrev.5b00623

Park E, Maquat LE. 2013. Staufen-mediated mRNA decay. Wiley Interdiscip Rev RNA 4: 423-435. doi:10.1002/wrna.1168

Park E, Gleghorn ML, Maquat LE. 2013. Staufen2 functions in Staufen1-mediated mRNA decay by binding to itself- and its paralog and promoting UPF1 helicase but not ATPase activity. Proc Natl Acad Sci USA 110: 405-412. doi:10.1073/pnas .1213508110

Peredo J, Villacé P, Ortín J, de Lucas S. 2014. Human Staufen1 associates to miRNAs involved in neuronal cell differentiation and is required for correct dendritic formation. PLoS ONE 9: e113704. doi:10.1371/journal.pone.0113704

Ramasamy S, Wang H, Quach HN, Sampath K. 2006. Zebrafish Staufen 1 and Staufen2 are required for the survival and migration of primordial germ cells. Dev Biol 292: 393-406. doi:10.1016/j .ydbio.2006.01.014

Rambo RP. 2017. Considerations for sample preparation using sizeexclusion chromatography for home and synchrotron sources. AdvExp Med Biol 1009:31-45. doi:10.1007/978-981-10-6038-0_3

Ramos A, Bayer P, Varani G. 1999. Determination of the structure of the RNA complex of a double-stranded RNA-binding domain from Drosophila Staufen protein. Biopolymers 52: 181-196. doi: 10.1002/1097-0282(1999)52:4<181::AID-BIP1003>3.0.CO;2-5

Ramos A, Grünert S, Adams J, Micklem DR, Proctor MR, Freund S, Bycroft M, St Johnston D, Varani G. 2000. RNA recognition by a Staufen double-stranded RNA-binding domain. EMBO J 19: 997-1009. doi:10.1093/emboj/19.5.997

Ravel-Chapuis A, Bélanger G, Yadava RS, Mahadevan MS, DesGroseillers L, Côté J, Jasmin BJ. 2012. The RNA-binding protein Staufen 1 is increased in DM1 skeletal muscle and promotes alternative premRNA splicing. J Cell Biol 196: 699-712. doi:10 $.1083 / j c b .201108113$
Ravel-Chapuis A, Crawford TE, Blais-Crépeau M-L, Bélanger G, Richer CT, Jasmin BJ. 2014. The RNA-binding protein Staufen1 impairs myogenic differentiation via a c-myc-dependent mechanism. Mol Biol Cell 25: 3765-3778. doi:10.1091/mbc.e14-040895

Ravel-Chapuis A, Klein Gunnewiek A, Bélanger G, Crawford Parks TE, Côté J, Jasmin BJ. 2016. Staufen 1 impairs stress granule formation in skeletal muscle cells from myotonic dystrophy type 1 patients. Mol Biol Cell 27: 1728-1739. doi:10.1091/mbc.e15-06-0356

Ricci EP, Kucukural A, Cenik C, Mercier BC, Singh G, Heyer EE, AsharPatel A, Peng L, Moore MJ. 2014. Staufen1 senses overall transcript secondary structure to regulate translation. Nat Struct Mol Biol 21: 26-35. doi:10.1038/nsmb.2739

Rigbolt KT, Prokhorova TA, Akimov V, Henningsen J, Johansen PT, Kratchmarova I, Kassem M, Mann M, Olsen JV, Blagoev B. 2011. System-wide temporal characterization of the proteome and phosphoproteome of human embryonic stem cell differentiation. Sci Signal 4: rs3. doi:10.1126/scisignal.2001570

Schupbach T, Wieschaus E. 1986. Germline autonomy of maternal-effect mutations altering the embryonic body pattern of Drosophila. Dev Biol 113: 443-448. doi:10.1016/0012-1606(86)90179-X

St Johnston D, Beuchle D, Nusslein-Volhard C. 1991. Staufen, a gene required to localize maternal RNAs in the Drosophila egg. Cell 66: 51-63. doi:10.1016/0092-8674(91)90138-O

St Johnston D, Brown NH, Gall JG, Jantsch M. 1992. A conserved double-stranded RNA-binding domain. Proc Natl Acad Sci USA 89: 10979-10983. doi:10.1073/pnas.89.22.10979

Sugimoto Y, Vigilante A, Darbo E, Zirra A, Militti C, D'Ambrogio A, Luscombe NM, Ule J. 2015. hiCLIP reveals the in vivo atlas of mRNA secondary structures recognized by Staufen 1. Nature 26: 491-494. doi:10.1038/nature14280

Thomas JM, Beal PA. 2017. How do ADARs bind RNA? New proteinRNA structures illuminate substrate recognition by the RNA editing ADARs. Bioessays 39. doi:10.1002/bies.201600187

Tria G, Mertens HD, Kachala M, Svergun DI. 2015. Advanced ensemble modelling of flexible macromolecules using $X$-ray solution scattering. IUCrJ 2: 207-217. doi:10.1107/S205225251500202X

Tsutakawa SE, Lafrance-Vanasse J, Tainer JA. 2014. The cutting edges in DNA repair, licensing, and fidelity: DNA and RNA repair nucleases sculpt DNA to measure twice, cut once. DNA Repair (Amst) 19: 95-107. doi:10.1016/j.dnarep.2014.03.022

Vessey JP, Macchi P, Stein JM, Mikl M, Hawker KN, Vogelsang P, Wieczorek K, Vendra G, Riefler J, Tubing F, et al. 2008. A loss of function allele for murine Staufen1 leads to impairment of dendritic Staufen1-RNP delivery and dendritic spine morphogenesis. Proc Natl Acad Sci USA 105: 16374-16379. doi:10.1073/pnas 0804583105

Vranken WF, Boucher W, Stevens TJ, Fogh RH, Pajon A, Llinas M, Ulrich EL, Markley JL, lonides J, Laue ED. 2005. The CCPN data model for NMR spectroscopy: development of a software pipeline. Proteins 59: 687-696. doi:10.1002/prot.20449

Wang X, Vukovic L, Koh HR, Schulten K, Myong S. 2015. Dynamic profiling of double-stranded RNA binding proteins. Nucleic Acids Res 43: 7566-7576. doi:10.1093/nar/gkv726

Weigelt J. 1998. Single scan, sensitivity- and gradient-enhanced TROSY for multidimensional NMR experiments. J Am Chem Soc 120: 10778-10779. doi:10.1021/ja982649y

Wickham L, Duchaîne T, Luo M, Nabi IR, DesGroseillers L. 1999. Mammalian staufen is a double-stranded-RNA- and tubulin-binding protein which localizes to the rough endoplasmic reticulum. Mol Cell Biol 19: 2220-2230. doi:10.1128/MCB.19.3.2220

Zhou H, Di Palma S, Preisinger C, Peng M, Polat AN, Heck AJ, Mohammed S. 2013. Toward a comprehensive characterization of a human cancer cell phosphoproteome. J Proteome Res 12: 260-271. doi:10.1021/pr300630k 

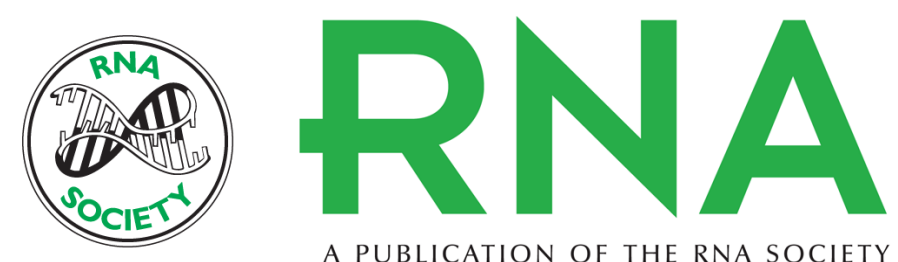

A PUBLICATION OF THE RNA SOCIETY

\section{A multipronged approach to understanding the form and function of hStaufen protein}

Silvia Visentin, Giuseppe Cannone, James Doutch, et al.

RNA 2020 26: 265-277 originally published online December 18, 2019

Access the most recent version at doi:10.1261/rna.072595.119

\section{Supplemental http://rnajournal.cshlp.org/content/suppl/2019/12/18/rna.072595.119.DC1 Material}

References This article cites 69 articles, 27 of which can be accessed free at: http://rnajournal.cshlp.org/content/26/3/265.full.html\#ref-list-1

Creative This article is distributed exclusively by the RNA Society for the first 12 months after the Commons License full-issue publication date (see http://rnajournal.cshlp.org/site/misc/terms.xhtml). After 12 months, it is available under a Creative Commons License (Attribution-NonCommercial 4.0 International), as described at http://creativecommons.org/licenses/by-nc/4.0/.
Email Alerting Receive free email alerts when new articles cite this article - sign up in the box at the Service top right corner of the article or click here.

\section{|||||||| Providing Precise Solutions for your research.}

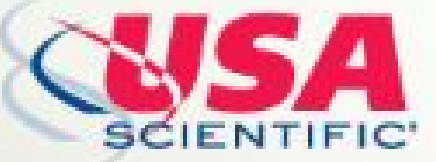

To subscribe to $R N A$ go to:

http://rnajournal.cshlp.org/subscriptions

(C) 2020 Visentin et al.; Published by Cold Spring Harbor Laboratory Press for the RNA Society 